\title{
Antimycobacterial, antiplasmodial studies and cytotoxicity of oleanolic acid and its derivative from Syzygium aromaticum Linn (Myrtaceae)
}

\author{
Estudos antiplasmódicos, antimicobacterianos e citotoxicidade do ácido oleanólico e do seu \\ derivado da Syzygium aromaticum Linn (Myrtaceae)
}

\author{
V. O. Fadipe ${ }^{1,2^{*}}$, A. R. Opoku ${ }^{2}$, M. Singh ${ }^{3,4}$, A. R. Pereira ${ }^{5}$, P. Rijo ${ }^{5}$, N. I. Mongalo ${ }^{6}$
}

${ }^{1}$ Department of Chemistry, University of Zululand, Private Bag X1001,KwaDlangezwa 3886, South Africa; ${ }^{2}$ Federal Ministry of Science and Technology, Abuja, Nigeria; ${ }^{3}$ Department of Biochemistry \& Microbiology, University of Zululand, Private Bag X1001, KwaDlangezwa 3886, South Africa; ${ }^{4}$ Discipline of Biochemistry, University of KwaZulu-Natal, Private Bag X54001, Durban 4000, South Africa; ${ }^{5} \mathrm{CBIOS}$ - Research Center for Biosciences and Health Techonologies, Campo Grande 376, Lisbon, Portugal; ${ }^{6}$ College of Agriculture and Environmental Sciences (CAES) Laboratories, University of South Africa, Private Bag X6, Florida, Johannesburg, South Africa

*corresponding author: vobfadipe@yahoo.com

\begin{abstract}
Malaria and tuberculosis are among the most common death causes and threats to human life globally. In a quest to find new antimalarial and anti-mycobacterial drugs, oleanolic acid (OA) was isolated from the flower buds of Syzygium aromaticum Linn (Myrtaceae). The derivative, 3-O-Acetyl-Oleanolic acid (OAA), was synthesized from the isolated product and the biological activities of the two compounds were compared. The antiplasmodial and antimycobacterial activity of oleanolic acid and its derivative were investigated against Plasmodium falciparum (Chloroquine Sensitive Strain) NF54 and Mycobacterium tuberculosis H37Rv. OAA exhibited IC50 of $4.3 \mu \mathrm{g} / \mathrm{ml}$ against $P$. falciparum, compared with an $\mathrm{IC}_{50}$ of $27.4 \mu \mathrm{g} / \mathrm{ml}$ for OA, while against $M$. tuberculosis OAA showed $0.1 \mu \mathrm{g} / \mathrm{ml}$, compared with an $\mathrm{IC}_{50}$ of $0.042 \mu \mathrm{g} / \mathrm{ml}$ for OA. In the cytotoxicity studies, using the MTT assay, both compounds exhibited LC50 of $>300$ $\mu \mathrm{g} / \mathrm{ml}$ against both human embryonic kidney (HEK293) and hepatocellular carcinoma cell lines (HepG2), suggesting that the compounds are not cytotoxic at low to moderate concentrations against the tested cell lines. In conclusion, the derivative OAA improved the antiplasmodial activity but no improvement was shown for antimycobacterial activity, in comparison with the starting material OA. Thus, more derivatives could be designed, using OA as starting material, to obtain future antiplasmodial compounds.
\end{abstract}

Keywords: Oleanolic acid, Syzygium aromaticum, cytotoxicity, antimycobacterial, antiplasmodial activity

\section{Resumo}

A malária e a tuberculose são um risco de saúde mundial responsáveis por muitas mortes. Na procura de novos fármacos, o ácido oleanólico (OA) foi isolado a partir dos botões das flores de Syzygium aromaticum. O seu derivado, o ácido 3-O-Acetil-oleanólico (OAA) foi sintetizado a partir do produto isolado e as respectivas atividades biológicas comparadas. As atividades antiplasmódicas e antimicobaterianas do AO e do OAA foram estudadas contra o Plasmodium falciparum (sensível à cloroquina) NF54 e Mycobacterium tuberculosis H37Rv respetivamente. O OAA apresentou um $\mathrm{IC}_{50}$ de $4.3 \mu \mathrm{g} / \mathrm{ml}$ contra P. falciparum em comparação com o IC50 de $27.4 \mu \mathrm{g} / \mathrm{ml}$ para o OA; quando testado contra M. Tuberculosis, o OAA apresentou um IC50 de $0,1 \mu \mathrm{g} / \mathrm{ml}$, em comparação com IC s0 $_{50}$ de $042 \mu \mathrm{g} / \mathrm{ml}$ para OA. Nos estudos de citotoxicidade (MTT) ambos apresentaram LC50>300 $\mu \mathrm{g} / \mathrm{ml}$ contra células embrionárias renais humanas (HEK293) e linhagens de carcinoma hepatocelular (HepG2), sugerindo que os compostos não são tóxicos em concentrações baixas a moderadas nas células em estudo. Em conclusão, o OAA melhorou a atividade antiplasmodial, sem aumento da atividade antimicobacteriana, em comparação com o OA. Mais derivados poderiam ser desenhados, usando OA como material de partida, para obter futuros compostos antiplasmodiais.

Palavras-chave: Ácido oleanólico, Syzygium aromaticum, citotoxicidade, atividade antiplasmódica 


\section{Introduction}

Tuberculosis (TB) and malaria are diseases that have emerged as serious pandemics against humanity. In conjunction with malaria, TB is ranked as the second leading cause of death from a contagious disease after HIV-AIDS (1). Both are opportunistic diseases accompanying immunocompromised patients. Malaria is a parasitic infection caused by the bites of Anopheles mosquitoes infected with Plasmodium species such as Plasmodium falciparum, Plasmodium vivax, Plasmodium malariae and Plasmodium ovale (2), while TB is a pulmonary infection caused by a bacterium Mycobacterium tuberculosism (3) and may co-infect humans with HIV and result in various forms of TB including multidrug resistant TB (MDR-TB), extensively drug resistant TB (XDR-TB), and completely-drug resistant TB (TDR-TB). As a result, several efforts must put in place to prevent, manage and treat these diseases through the development of vaccines and new antibiotics to help current preventatives and therapies (4-6) and the cost of relevant antimalarial and anti-tubercular drugs is extremely high (7). Medicinal and food plants could therefore be scrutinized for new potent therapeutic agents against malaria and TB.

Syzygium aromaticum, commonly known as "Cloves" is an evergreen tree belonging to family Myrtaceae and its flower bud is commonly used as a spice (8). Besides being used as a condiment in foods, cloves may be used in the treatment of toothache, mouth pain, malaria, tuberculosis, cholera, and throat inflammation (9). The extracts and essential oils from the plant species are known to possess antifungal activity against Penicillium citrinum, Paecilomycetes variotii and Aspergillus niger (10-13), and antibacterial activity against some food and human pathogens which includes Staphylococcus aureus, Salmonella typhimurium, Escherichia coli, Shigella spp, Vibrio spp, Pseudomonas aeruginosa and Clostridium botulinum (14-17). Moreover, the extracts are also known to contain antioxidant, anti-viral, larvicidal, anti-platelet, anti-stress, anti-pyretic, anesthetic, anti-cancer anti-inflammatory, anti-diabetic, actinoceptive and insecticidal activity amongst other pharmacological activities. $(18,19)$ The current paper aimed to investigate the anti-mycobacterial and anti-plasmodial properties of oleanolic acid (OA) and its derivative against Mycobacterium tuberculosis and Plasmodium falciparum, respectively. Furthermore, the cytotoxicity of such compounds against the human embryonic kidney (HEK293) and human hepatocellular carcinoma (HepG2) cells were also evaluated.

\section{Introdução}

A tuberculose (TB) e a malária são doenças que emergiram como uma séria pandemia contra a Humanidade. Em conjunto com a malária, a TB é classificada como a segunda maior causa de morte proveniente de uma doença contagiosa a seguir ao HIV-AIDS (1). Ambas são doenças oportunistas comuns em indivíduos imunocomprometidos. A malária é uma infeção parasítica causada pela picada do mosquito Anopheles infetado com um parasita da espécie Plasmodium tais como Plasmodium falciparum, Plasmodium vivax, Plasmodium malariae e Plasmodium ovale (2), enquanto a TB é uma infeção pulmonar causada pela bactéria Mycobacterium tuberculosis (3). Esta pode co-infetar humanos com HIV e resultar em varias formas de TB incluindo tuberculose multirresistente a fármacos (MDR-TB), TB extensamente resistente a fármacos (XDR-TB) e TB totalmente resistente a fármacos (TDR-TB). Por esse motivo, vários esforços estão a ser feitos de modo a prevenir, gerir e tratar estas doenças através do desenvolvimento de vacinas e novos antibióticos de modo a modo a diminuir a pressão nos atualmente utilizados (4-6), sendo o custo dos fármacos antimaláricos e anti-tuberculosos relevantes é extremamente alto (7). Portanto, as plantas medicinais e alimentares podem ser estudadas na procura de novos agentes contra a malária e a TB.

Syzygium aromaticum conhecido como Cravo-da-Índia é uma árvore perene pertencente à família Myrtaceae e a sua flor é comumente utilizada como especiaria (8). Além de ser utilizada como um condimento na alimentação, pode também ser utilizada como um tratamento na dor de dentes, boca, tuberculose malárica, cólera e inflamação da garganta (9). Os extratos e óleos essenciais desta planta são conhecidos por apresentarem propriedades antifúngicas contra Penicillium citrinum, Paecilomycetes variotii and Aspergillus niger (10-13) e atividade antibacteriana contra alguns patogénicos alimentares e humanos incluindo Staphylococcus aureus, Salmonella typhimurium, Escherichia coli, Shigella spp, Vibrio spp, Pseudomonas aeruginosa and Clostridium botulinum (14-17). Além disso os extratos também são conhecidos por conter atividades antioxidante, antiviral, antisstress, antipirética, anestética, anticancerígena, anti-inflamatória, antidiabética, actinoceptiva, inseticida, entre outras atividades farmacológicas $(18,19)$. O atual estudo tem como objetivo investigar as propriedades antimicobacterianas e antiplasmódicas do ácido oleanólico e do seu derivado contra Mycobacterium tuberculosis e Plasmodium falciparum respetivamente. Este estudo também tem como objetivo avaliar 


\section{Materials and Methods}

The Syzygium aromaticum flower buds were purchased from an Indian spice shop in Richard Bay, South Africa. The buds were properly authenticated by a plant botanist and a herbarium curator in the Department of Botany, University of Zululand. South Africa.

\section{Isolation of oleanolic acid (OA)}

The plant Syzigum aromaticum $(2.0 \mathrm{~kg}$ ) was macerated in ethyl acetate twice, filtered through Whatman $\mathrm{N}^{\mathrm{o}} 1$ filter paper and concentrated using a rotary evaporator (Bibby Scientific Limited/Cole-Parmer, Staffordshire, UK). The ethyl acetate extract was defatted twice with $n$-hexane and the solid residue was collected (9.6 g). $5.2 \mathrm{~g}$ of defatted ethyl acetate extract was then packed into the top of the column containing silica gel $60(6 \mathrm{x}$ $120 \mathrm{~cm}$ ) and eluted with $n$-hexane, and then ethyl acetate: $n$-hexane (3:7) to afford a white amorphous solid $(385 \mathrm{mg})$ which was identified as oleanolic acid (OA) after recrystallization from $\mathrm{MeOH}$.

\section{Preparation of 3-O-Acetyl-Oleanolic acid}

A mixture of oleanolic acid $(250 \mathrm{mg})$, acetic acid anhydride $(10 \mathrm{ml})$, and pyridine $(3 \mathrm{ml})$ was stirred at $40{ }^{\circ} \mathrm{C}$ for 6 hours. The mixture was transferred into $500 \mathrm{ml}$ of distilled water and stirred continuously for 2 hours at room temperature for hydrolysis. It was then filtered, dried, and washed with $50 \mathrm{ml}$ dilute hydrochloric acid $(10 \%)$ to remove any traces of pyridine. The residue was dried and packed into the top of a small $(2.5 \times 40$ $\mathrm{cm})$ column and then eluted with ethyl acetate: $n$-hexane (1:9) and after evaporation afford a white amorphous powder $(112 \mathrm{mg})$ which was purified by recrystallization from $\mathrm{MeOH}$.

\section{Spectroscopic analysis}

Nuclear magnetic resonance (NMR): ${ }^{1} \mathrm{H}-,{ }^{13} \mathrm{C}-\mathrm{NMR}$ and all 2D spectra were recorded on an Avance NMR instrument (Bruker Daltonics, Bremen, Germany) operating at $400 \mathrm{MHz}$. Chemical shifts are reported as $\delta$ values (ppm) relative to an internal standard of tetramethylsilane (TMS) or to the solvent line of $\mathrm{CDCl}_{3}$ $\left({ }^{8} \mathrm{H}=7.26 \mathrm{ppm},{ }^{8} \mathrm{C}=77.16 \mathrm{ppm}\right)$. a citotoxicidade destes compostos em células renais embrionárias humanas (HEK293) e células humanas de carcinoma hepatocelular (HepG2).

\section{Materiais e Métodos}

Os botões das flores de Syzygium aromaticum foram adquiridos numa loja de especiarias indiana em Richard Bay, África do Sul. O material vegetal foi autenticadas por um botânico e um curador de herbário do Departamento de Botânica da Universidade de Zululand, África do Sul.

\section{Isolamento do ácido oleanólico}

A planta Syzigum aromaticum $(2.0 \mathrm{~kg})$ foi macerada em acetato de etilo duas vezes, filtrada através de papel de filtro Whatman $\mathrm{N}^{\circ} 1$ e concentrada no evaporador rotativo (Bibby Scientific Ltd/Cole-Parmer, Staffordshire, GB). O acetato de etilo extraído foi lavado duas vezes com $n$-hexano e os resíduos sólidos recolhidos $(9,6 \mathrm{~g})$. 5,2 $\mathrm{g}$ do extrato de acetato de etilo foram depois colocadas numa coluna com $(6 \times 120 \mathrm{~cm})$ sílica gel 60 e eluídas com $n$-hexano, e posteriormente com acetato de etilo: $n$-hexano (3:7) de modo a obter um solido branco amorfo (385 mg) que foi identificado como ácido oleanólico (OA) após recristalização de metanol.

\section{Preparação de ácido 3-O-Acetil-Oleanólico}

Uma mistura de ácido oleanólico (250 mg), ácido acético anidro $(10 \mathrm{ml})$ e piridina $(3 \mathrm{ml})$ foi agitada a 40 ${ }^{\circ} \mathrm{C}$ durante 6 horas. A mistura foi transferida para 500 $\mathrm{ml}$ de água destilada e agitada continuamente durante 2 horas à temperatura ambiente para hidrolisar. Foi então filtrada, seca e lavada com $50 \mathrm{ml}$ com ácido clorídrico diluído $(10 \%)$ para eliminar quaisquer resíduos de piridina. O resíduo foi seco e colocado numa pequena coluna $(2.5 \times 40 \mathrm{~cm})$ e eluído com uma mistura de acetato de etilo: $n$-hexano (1:9) e após evaporação obteve-se um pó branco e amorfo (112 mg) que foi purificado por recristalização de metanol.

\section{Análise espectroscópica}

Ressonância magnética nuclear (RMN): ${ }^{1} \mathrm{H}-,{ }^{13} \mathrm{C}-\mathrm{NMR}$ e todos os espectros 2D foram realizados num equipamento Avance a $400 \mathrm{MHz}$ (Bruker Daltonic, Bremen, FRD) os desvios químicos foram reportados como valores $\delta$ (ppm) relativamente ao padrão interno do tetrametilsilano (TMS) ou relativamente à linha de solvente $\mathrm{CDCl}_{3}\left({ }^{\delta} \mathrm{H}=7,26 \mathrm{ppm},{ }^{\delta} \mathrm{C}=77,16 \mathrm{ppm}\right)$. 
High-resolution-mass spectrometry (HR-MS): Highresolution mass data were obtained using a microTOF-Q II ESI MS instrument (Bruker) operating at ambient temperature.

Infrared (IR): The infrared (IR) spectroscopy determination was carried out using a Spectrum 100 FTIR spectrometer (Perkin-Elmer, Waltham, MA, USA).

Melting point (mp): Melting points of the compounds were determined on an SMP3 apparatus (Stuart Scientific/Cole-Parmer, Staffordshire UK).

\section{Biological assays}

\section{Antimycobacterial evaluation}

\section{Bacterial strains for anti-TB biological assay}

For the preparation of the inoculum, a virulent strain of M. tuberculosis (H37Rv, ATCC 27294) bacteria was grown in $100 \mathrm{ml}$ of Middlebrook 7H9 Broth (Difco, Detroit, MI, USA), supplemented with $0.2 \%$ (v/v) glycerol (Sigma Chemical Co., St Louis, MO, USA), 10\% (v/v) OADC (oleic acid, albumin, dextrose, catalase; Difco) and $0.05 \%(\mathrm{v} / \mathrm{v})$ Tween 80 (Sigma).

\section{Microplate Alamar Blue Assay (MABA)}

Anti-TB susceptibility testing of the isolate and the synthesized product was determined using the fluorometric Microplate Alamar Blue Assay (MABA) as described previously.(20,21) The MICs against MTB H37RV (ATCC 27294) were assessed using rifampin and isoniazid as positive controls. Sample stocks were prepared in $5 \% \mathrm{DMSO}$, and two-fold dilutions of compounds were prepared in Middlebrook 7H12 medium (7H9 Broth containing $0.1 \% \mathrm{w} / \mathrm{v}$ casitone, $5.6 \mu \mathrm{g} / \mathrm{mL}$ palmitic acid, $5 \mathrm{mg} / \mathrm{mL}$ bovine serum albumin, $4 \mathrm{mg} / \mathrm{mL}$ catalase, filter-sterilized) in a volume of $100 \mu \mathrm{L}$ in 96-well microplates (black/clear flat bottom, BD Optilux, BD Biosciences, Billerica, MA, USA). MTB cultures (100 $\mu \mathrm{L}$ inoculum of $2 \times 10^{5} \mathrm{CFU} / \mathrm{mL}$ ) were added, yielding a final testing volume of $200 \mu \mathrm{L}$. The plates were incubated at $37{ }^{\circ} \mathrm{C}$. On the seventh day of incubation, 12.5 $\mu \mathrm{L}$ of $20 \%$ Tween 80 , and $20 \mu \mathrm{L}$ of Alamar Blue (Invitrogen BioSource/ThermoFisher Scientific, Waltham, MA, USA) were added to the wells. After incubation at $37{ }^{\circ} \mathrm{C}$ for $16-24 \mathrm{~h}$, the fluorescence of the wells was measured at excitation of $530 \mathrm{~nm}$ and emission of 590 $\mathrm{nm}$. The MIC was determined as the lowest concentration resulting in a reduction in fluorescence of $\geq 90 \%$ relative to the mean of replicate bacteria-only controls.
Espectrometria de massa de alta resolução (HR-MS): Os dados de alta resolução foram obtidos usando um equipamento micro TOF-Q ESI (Bruker) à temperatura ambiente.

Espectroscopia de Infravermelhos (IRS): A espectroscopia de infravermelhos foi realizada com o auxílio de um espectrómetro, Spectrum 100 FTIR, Perkin-Elmer,Waltham, MA, EUA

Ponto de fusão (mp):Os pontos de fusão dos compostos foram determinados num aparelho SMP3 (Stuart Scientmific/Cole-Parmer, Staffordshire GB).

\section{Ensaios Biológicos}

\section{Avaliação antimicobacteriana}

\section{Estirpes de bactérias para o ensaio biológico anti-TB}

Para a preparação do inóculo foi utilizada uma estirpe virulenta de M. tuberculosis (H37Rv, ATCC 27294), a bactéria cresceu em $100 \mathrm{ml}$ de Middlebrook 7H9 Broth (Difco, Detroit, MI, EUA), com um suplemento de $0,2 \%$ (v/v) glicerol (Sigma Chemical Co., St Louis, MO, EUA), 10\% (v/v) OADC (ácido oleico, albumina, dextrose, catalase; Difco) e $0.05 \%$ (v/v) Tween 80 (Sigma).

\section{Ensaio Azul de Alamar em microplacas (MABA)}

A suscetibilidade anti-TB do isolado e do produto sintetizado foi determinada usando o ensaio fluorométrico Azul de Alamar em microplacas como descrito anteriormente $(20,21)$. Os valores de MIC contra MTB H37RV (ATCC 27294) foram avaliados usando a rifampina e a isoniazida como controlos positivos. As amostras foram preparadas em 5\% DMSO e foram realizadas duas diluições dos compostos em meio Middlebrook 7H12 (7H9 caldo contendo $0.1 \% \mathrm{w} / \mathrm{v}$ casitona, $5.6 \mu \mathrm{g} / \mathrm{mL}$ acido palmitico, $5 \mathrm{mg} / \mathrm{mL}$ albumina de sérum bovino, $4 \mathrm{mg} / \mathrm{mL}$ catalase, esterilizada por filtro) num volume de $100 \mu \mathrm{L}$ numa microplaca de 96 poços (fundo liso pretoltransparente, BD Optilux, BD Biosciences, Billerica, MA, EUA). Culturas MTB (100 $\mu \mathrm{L}$ inoculo of $2 \times 10^{5} \mathrm{CFU} / \mathrm{mL}$ ) foram adicionadas, obtendo um volume final de $200 \mu \mathrm{L}$. As placas foram incubadas a $37{ }^{\circ} \mathrm{C}$. Ao fim de sete dias de incubação foram adicionados aos poços $12.5 \mu \mathrm{L}$ de $20 \%$ Tween 80 , and $20 \mu \mathrm{L}$ de azul Alamar (Invitrogen BioSource/ ThermoFisher Scientific, Waltham, MA, EUA). Após 16-24 h de incubação a $37^{\circ} \mathrm{C}$ a fluorescência dos poços foi medida excitation at $530 \mathrm{~nm}$ and emission at 590 nm. O MIC foi determinado como a concentração 


\section{In vitro antiplasmodial activity}

The test samples, the isolate, and the chemical derivative were tested in triplicate against a chloroquine-sensitive strain (CQS) of Plasmodium falciparum (D10). Continuous in vitro cultures of asexual erythrocyte stages of $P$. falciparum were maintained using a modified method as reported by Trager and Jensen et al (22). Quantitative assessment of anti-plasmodial activity was made in vitro using the parasite lactate dehydrogenase assay as described by Makler et al (23). The test samples were prepared as a $20 \mathrm{mg} / \mathrm{mL}$ stock solution in $10 \%$ DMSO and sonicated to enhance solubility. Stock solutions were stored at $-20^{\circ} \mathrm{C}$. Further dilutions were prepared on the day of the experiment. Chloroquine and artesunate were used as reference drugs. A full dose-response was performed for all active compounds to determine the initial concentration inhibiting $50 \%$ of parasite growth $\left(\mathrm{IC}_{50}\right.$ value). Test samples were evaluated at a starting concentration of $100 \mathrm{ug} / \mathrm{mL}$, which was then serially diluted two-fold in complete medium to give ten preparations of variable concentrations, with the lowest concentration being $0.2 \mu \mathrm{g} / \mathrm{mL}$. The same dilution technique was used for all samples. The chloroquine (CQ) was tested at the starting concentration of $1000 \mathrm{ng} / \mathrm{mL}$. The highest concentration of solvent to which the parasite-infected erythrocytes were exposed to had no measurable effect on the parasite viability (data not shown). The $\mathrm{IC}_{50}$ values were obtained using a non-linear dose-response curve fitting analysis via GraphPad Prism v.4.0 software (GraphPad Prism, Inc; San Diego, CA, USA). IC $_{50}$ was defined as the $50 \%$ concentration of test compound that exhibited inhibition of the parasite.

\section{Cytotoxicity studies}

\section{MTT Cell Proliferation Assay}

Human embryonic kidney (HEK293) and human hepatocellular carcinoma (HepG2) cells were all grown to confluency in $25 \mathrm{~cm}^{3}$ flasks. Cells were then trypsinized and plated into 48 well plates at specific seeding densities. Cells were incubated overnight at $37{ }^{\circ} \mathrm{C}$. Medium was then removed and fresh medium (MEM + Glutmax + antibiotics) was added. Isolates and synthesized compounds $(50-350 \mu \mathrm{g} / \mathrm{mL})$ were then added in triplicate and incubated for $4 \mathrm{~h}$. The medium then was removed and replaced with complete medium (MEM + Glutmax mais baixa eficaz em reduzir a fluorescência a $\geq$ $90 \%$ relativamente à média dos controlos replicados contendo apenas bactéria.

\section{Atividade antiplasmódica in vitro}

As amostras em teste, o isolado $\mathrm{AO}$ e o derivado químico OAA, foram testadas em triplicado contra uma estirpe de Plasmodium falciparum (D10) sensível à cloroquina (CQS). Culturas continuas in vitro de estádios de eritrócitos assexuais de $P$. falciparum foram mantidas usando o método modificado de Trager e Jensen et al. (22). A analise quantitativa de atividade antiplasmódica foi realizada $n$ vitro utilizando o ensaio da lactato desidrogenase parasítica como descrito por Makler et al (23). A amostra foi preparada numa concentração de $20 \mathrm{mg} / \mathrm{mL}$ numa solução-mãe em 10\% DMSO e foi realizada a sonificação de modo a melhorar a sua solubilidade. A solução-mãe foi armazenada a $-20^{\circ} \mathrm{C}$. As diluições posteriores foram preparadas no dia do ensaio. A cloroquina e o artenusato foram usadas como os fármacos de referência para os ensaios. Uma resposta total ao fármaco foi realizada para todos os compostos ativos de modo a determinar a concentração inicial inibitória de $50 \%$ do crescimento do parasita (valor $\mathrm{IC}_{50}$ ). As amostras foram testadas a uma concentração inicial de $100 \mu \mathrm{g} / \mathrm{mL}$, que foi posteriormente diluído em serie duas vezes em meio para obter dez preparações de variadas concentrações; sendo a concentração mais baixa $0,2 \mu \mathrm{g} / \mathrm{mL}$. Foi utilizada a mesma técnica de diluição para todas as amostras. CQ foi testada com a concentração inicial de $1000 \mathrm{ng} / \mathrm{ml}$. A concentração mais alta de solvente na qual o os eritrócitos infetados por parasitas foram expostos não teve efeito mesurável na viabilidade do parasita (dados não apresentados). Os valores de $\mathrm{IC}_{50}$ foram obtidos usando uma análise de curva não- linear de fármaco-resposta através de Graph pad Prism v.4.0 software (Graph Pad Prism, Inc; San Diego, CA,

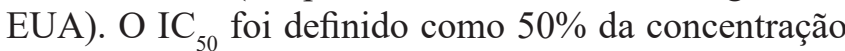
de amostra que apresentou inibição do parasita.

\section{Estudos de citotoxicidade}

\section{Ensaio de proliferação celular MTT}

Células humanas renais embrionárias (HEK293) e de carcinoma hepatocelular humano (HepG2) foram cultivadas em frascos de $25 \mathrm{~cm}^{3}$. Foram então tripsinizadas e plaqueadas em placas de 48 poços com densidades de semeação especificas. As células foram incubadas durante a noite a $37{ }^{\circ} \mathrm{C}$. O meio foi removido e foi adicionado meio fresco (MEM + Glutmax + antibióticos). Os isolados e compostos sintetizados $(50-350 \mu \mathrm{g} / \mathrm{mL})$ 
+ antibiotics $+10 \%$ Fetal bovine serum). After 48 h, cells were subjected to the MTT assay (24). Data was evaluated through regression analysis using QED statistics program and from the linear equation the $\mathrm{IC}_{50}$ values representing the lethal concentration for $50 \%$ mortality was calculated.

\section{Results and Discussion}

Oleanolic acid is a triterpene compound with pentacyclic cholesterol-like structure with low water solubility widely found in food, medicinal herbs and other plants $(25,26)$. The ${ }^{1} \mathrm{H}-$ and ${ }^{13} \mathrm{C}-\mathrm{NMR}$ data of both oleanolic acid (OA) and 3-O-acetyl-oleanolic acid (OAA) are summarized in Table 1. The synthesis of the derivative OAA is shown in Figure 1, and the anti-mycobacterial, anti-plasmodial, and the cytotoxicity properties of the two compounds are shown in Table 2.

\section{Oleanolic acid}

The white powder which was obtained after recrystallization in $\mathrm{MeOH}$ had a melting point of $299-301^{\circ} \mathrm{C}$. The infra-red spectra shows absorption at $3384 \mathrm{~cm}^{-1}$, indicating $(\mathrm{O}-\mathrm{H})$ stretch for hydroxyl hydrogen bond, while the signal at $1686 \mathrm{~cm}^{-1}$ is due to the presence of $(\mathrm{C}=\mathrm{O}$, acid) stretch of a carbonyl carbon, which suggests the presence of carboxylic acid $(\mathrm{COOH})$ functional group. The signal at $2937 \mathrm{~cm}^{-1}$ is due to $(\mathrm{C}-\mathrm{H})$ stretching for highly saturated alkane molecule. Proton decoupled ${ }^{13} \mathrm{C}-\mathrm{NMR}$ spectrum shows 30 carbon atoms (Table 1). The DEPT-90 sub spectrum showed five methane $(\mathrm{CH})$ carbons while DEPT-135 suggested ten (10) methylene foram adicionados em triplicado e incubados durante 4 h. Posteriormente o meio foi removido e foi adicionado um meio completo (MEM + Glutmax + antibióticos + $10 \%$ sérum bovino fetal). Apos $48 \mathrm{~h}$ as células foram sujeitas ao ensaio MTT (24). Os dados foram avaliados através de regressão linear usando o programa estatístico QED e através da equação linear os valores de $\mathrm{IC}_{50}$ representando uma concentração letal com $50 \%$ de mortalidade foram calculados.

\section{Resultados e Discussão}

O ácido oleanólico é um composto triterpénico com uma estrutura pentacíclica semelhante ao colesterol e com baixa solubilidade em água, que ocorre vastamente na alimentação, ervas medicinais e outras plantas $(25,26)$. Os dados de RMN do ácido oleanólico (OA) e do ácido 3-O-acetil-oleanólico (OAA) estão apresentados na Tabela 1. A síntese do derivado OAA está apresentado na Figura 1 e as propriedades antimicobacterianas, antiplasmódicas e citotóxicidade de ambos os compostos estão apresentadas na Tabela 2.

\section{Ácido oleanólico (AO)}

O pó branco obtido após a recristalização em metanol apresenta um ponto de fusão entre $299-301{ }^{\circ} \mathrm{C}$. O espetro de infravermelho mostra uma absorção a $3384 \mathrm{~cm}^{-1}$ indicando assim um alargamento da ligação hidroxilo $(\mathrm{O}-\mathrm{H})$, enquanto que o sinal a $1686 \mathrm{~cm}^{-1}$ é devido à presença de um alargamento de um grupo carbonilo $(\mathrm{C}=\mathrm{O}$, acido), ambos estes dados provavelmente confirmam a presença de um ácido carboxílico $(\mathrm{COOH})$. O sinal da $2937 \mathrm{~cm}^{-1}$ é devido ao alongamento (C-H) de uma molécula alcano altamente saturada. O desacoplamento de protão do espectro ${ }^{13} \mathrm{C}$-NMR mostra 30 átomos de
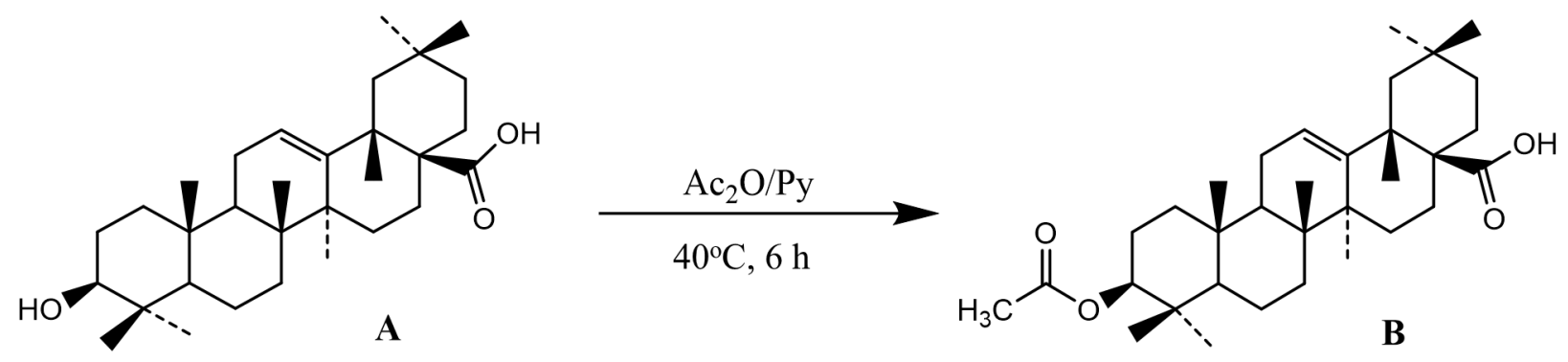

Figure 1 - Synthesis of 3-O-Acetyl-oleanolic acid(B) from oleanolic acid (A) acid. Py = pyridine

Figura 1 - Sintese de ácido 3-O-Acetil-oleanólico (B) apartir do ácido oleanólico (A). Py = piridina 
Table 1 - ${ }^{1} \mathrm{H}$ - and ${ }^{13} \mathrm{C}-\mathrm{NMR}$ obtained for oleanolic acid (OA) and for 3-0-acetyl-oleanolic acid (OAA)

Tabela 1 - Dados de $1 \mathrm{H}$ - e ${ }^{13} \mathrm{C}-\mathrm{RMN}$ obtidos do ácido oleanólico (OA) e do ácido 3-0-acetil-oleanólico (OAA)

\begin{tabular}{|c|c|c|c|c|c|}
\hline $\begin{array}{l}\text { Carbon Position / } \\
\text { Posição do } \\
\text { Carbono }\end{array}$ & $\begin{array}{c}{ }^{1} \mathrm{H}-\mathrm{NMR} \\
\text { OA (ppm) }\end{array}$ & $\begin{array}{c}{ }^{13} \mathrm{C} \text { NMR } \\
\text { OA }(\sigma)\end{array}$ & $\begin{array}{c}\text { C Type / } \\
\text { Tipo de C }\end{array}$ & $\begin{array}{c}{ }^{1} \mathrm{H} \text { NMR-OAA } \\
(\mathrm{ppm})\end{array}$ & $\begin{array}{c}{ }^{13} \mathrm{C} \text { NMR- } \\
\text { OAA }(\sigma)\end{array}$ \\
\hline 1 & & 38.6 & $\mathrm{CH}_{2}$ & & 39.4 \\
\hline 2 & & 27.3 & $\mathrm{CH}_{2}$ & & 23.7 \\
\hline 3 & $3.22(\mathrm{dd}, 4.2 / 4.96)$ & 79.2 & $\mathrm{CH}$ & $4.5(\mathrm{t})$ & 81.1 \\
\hline 4 & & 38.9 & $\mathrm{C}$ & & 37.97 \\
\hline 5 & & 55.3 & $\mathrm{CH}$ & & 55.4 \\
\hline 6 & & 18.5 & $\mathrm{CH}_{2}$ & & 16.98 \\
\hline 7 & & 33.2 & $\mathrm{CH}_{2}$ & & 33.94 \\
\hline 8 & & 39.4 & $\mathrm{C}$ & & 38.2 \\
\hline 9 & & 47.7 & $\mathrm{CH}$ & & 47.7 \\
\hline 10 & & 37.2 & $\mathrm{C}$ & & 37.1 \\
\hline 11 & & 23.1 & $\mathrm{CH}_{2}$ & & 23.5 \\
\hline 12 & $5.28(\mathrm{br})$ & 122.8 & $\mathrm{CH}$ & $5.55(\mathrm{br})$ & 122.7 \\
\hline 13 & & 143.7 & $\mathrm{C}$ & & 143.7 \\
\hline 14 & & 41.8 & $\mathrm{C}$ & & 41.7 \\
\hline 15 & & 27.8 & $\mathrm{CH}_{2}$ & & 26.0 \\
\hline 16 & & 23.5 & $\mathrm{CH}_{2}$ & & 32.6 \\
\hline 17 & & 46.6 & $\mathrm{C}$ & & 46.0 \\
\hline 18 & $2.82(\mathrm{~d}, 13.7)$ & 41.2 & $\mathrm{CH}$ & $2.8(\mathrm{~d}, 13.8)$ & 41.7 \\
\hline 19 & & 46.0 & $\mathrm{CH}_{2}$ & & 46.7 \\
\hline 20 & & 31.0 & $\mathrm{C}$ & & 32.6 \\
\hline 21 & & 34.0 & $\mathrm{CH}_{2}$ & & 33.2 \\
\hline 22 & & 32.6 & $\mathrm{CH}_{2}$ & & 28.0 \\
\hline 23 & $1.20(\mathrm{~s})$ & 28.3 & $\mathrm{CH}_{3}$ & $0.90(\mathrm{~s})$ & 23.7 \\
\hline 24 & $0.94(\mathrm{~s})$ & 15.7 & $\mathrm{CH}_{3}$ & $0.86(\mathrm{~s})$ & 18.3 \\
\hline 25 & $0.78(\mathrm{~s})$ & 15.5 & $\mathrm{CH}_{3}$ & $0.93(\mathrm{~s})$ & 15.5 \\
\hline 26 & $1.13(\mathrm{~s})$ & 17.5 & $\mathrm{CH}_{3}$ & $0.75(\mathrm{~s})$ & 16.8 \\
\hline 27 & $1.35(\mathrm{~s})$ & 26.1 & $\mathrm{CH}_{3}$ & $1.16(\mathrm{~s})$ & 23.7 \\
\hline 28 & & 182.6 & $\mathrm{C}$ & & 183.3 \\
\hline 29 & $0.91(\mathrm{~s})$ & 32.8 & $\mathrm{CH}_{3}$ & $0.85(\mathrm{~s})$ & 30.8 \\
\hline 30 & $0.98(\mathrm{~s})$ & 23.7 & $\mathrm{CH}_{3}$ & $0.92(\mathrm{~s})$ & 27.8 \\
\hline 31 & & & & & 171.2 \\
\hline 32 & & & & & 21.5 \\
\hline
\end{tabular}

Table 2 -Toxicity, anti-mycobacterial and anti-plasmodial activity of oleanolic acid (OA) and its derivative (OAA) Tabela 2 - Citotoxicidade, actividade anti-micobacteriana e anti-plasmodica do ácido oleanolico (OA) e do seu derivado (OAA)

\begin{tabular}{lcccc}
\hline $\begin{array}{l}\text { Compounds and controls / } \\
\text { Compostos e controlos }\end{array}$ & $\begin{array}{c}\text { Antimycobacterial } \\
\text { activity } \\
\text { Atividade } \\
\text { antimicobacteriana } \\
\left(\mathrm{IC}_{50} \mu \mathrm{g} / \mathrm{ml}\right)\end{array}$ & $\begin{array}{c}\text { Antiplasmodical } \\
\text { activity } / \\
\text { Actividade } \\
\text { antiplasmódica } \\
\left(\mathrm{IC}_{50} \mu \mathrm{g} / \mathrm{ml}\right)\end{array}$ & $\begin{array}{c}\text { Cytotoxicity / } \\
\text { Citotoxicidade } \\
\left(\mathrm{IC}_{50} \mu \mathrm{g} / \mathrm{ml}\right)\end{array}$ \\
\hline Oleanolic acid / & & & HEK293 & HepG2 \\
ácido Oleanólico (OA) & 0.042 & 27.4 & $>300$ & $>300$ \\
3-O-Acetyl-Oleanolic acid / & 0.100 & 4.3 & $>300$ & $>300$ \\
3-O-Acetil-Oleanólico (OAA) & $\mathrm{MIC}_{99}$ & $\mathrm{ng} / \mathrm{ml}$ & & \\
\hline & 0.01 & $\mathrm{ND}$ & & \\
\hline Rifampicina / Rifampicin & 3.125 & $\mathrm{ND}$ & & \\
Canamicina / Kanamycin & $\mathrm{ND}$ & 5.2 & & \\
Cloroquina / Chloroquine & $\mathrm{ND}$ & 2.1 & & \\
Artesunato / Artesunate & & & & \\
\hline
\end{tabular}


( $\mathrm{CH}_{2}$ negative), seven (7) methyl $\left(\mathrm{CH}_{3}\right.$, positive) and eight (8) quaternary carbon groups. The high resolution electron impact mass spectroscopy indicated molecular ion $[\mathrm{M}-\mathrm{H}]^{+}$at $\mathrm{m} / \mathrm{z} 457.7$, which is in agreement with the formula mass $456.7 \mathrm{~g} \mathrm{~mol}^{-1}$ for oleanolic acid.

\section{3-O-Acetyl-Oleanolic acid (OAA)}

The observed melting point of the white powder that was obtained after recrystallization in $\mathrm{MeOH}$ was 261-263 ${ }^{\circ} \mathrm{C}$. The infra-red spectra shows absorption at $3562 \mathrm{~cm}^{-1}$, indicating $(\mathrm{O}-\mathrm{H})$ stretch for hydroxyl hydrogen bond. The signal at $1767 \mathrm{~cm}^{-1}$ is as a result of carbonyl carbon $(\mathrm{C}=\mathrm{O}$, ester $)$, while the signal at 1729 $\mathrm{cm}^{-1}$ is due to the presence of $(\mathrm{C}=\mathrm{O}$, acid $)$ stretch of a carbonyl carbon, confirming the presence of carboxylic acid $(\mathrm{COOH})$ functional group. The signal at 2921 is due to the $(\mathrm{C}-\mathrm{H})$ stretch of a highly saturated alkane molecule. Proton decoupled ${ }^{13} \mathrm{C}-\mathrm{NMR}$ spectrum shows 32 carbon atoms (Table1). The DEPT-90 sub spectrum showed five methane $(\mathrm{CH})$ carbons, while DEPT-135 suggests ten (10) methylene $\left(\mathrm{CH}_{2}\right.$ negative), seven (7) methyl $\left(\mathrm{CH}_{3}\right.$, positive) and eight (8) quaternary carbon groups. The high resolution (electron impact) mass spectroscopy indicated molecular ion $[\mathrm{M}-\mathrm{H}]^{+}$at $\mathrm{m} / \mathrm{z}$ 499.37, which is in agreement with the formula mass $498.37 \mathrm{~g} / \mathrm{mol}$ for oleanolic acid acetate.

Several reports on the biological activities of $\mathrm{OA}$ are available in the literature, which include antiinflammatory, anti-oxidative, anti-HIV, antibacterial, and trypanocidal activity, with therapeutic efficacy studied against cancer, cardiovascular diseases, atherosclerosis, and diabetes (27). In the current study, the emphasis and focus are on the use of $\mathrm{OA}$ to synthesise and develop potent multi-target pharmacologically active lead compounds as active pharmaceutical ingredients against most common chronic immunity deficiency-related diseases. In the current work, OA exhibited potent anti-mycobacterial activity of $0.042\left(\mathrm{IC}_{50}\right.$ in $\left.\mu \mathrm{g} / \mathrm{ml}\right)$, which is more than two times effective than its derivative. This result is in agreement with other triterpenes such as betulinic acid, betulin, lupenone, and betulone from Alnus incana (Betulaceae) which exhibited $\mathrm{IC}_{50}$ ranging from 2.4 to $>100 \mu \mathrm{g} / \mathrm{ml}$ against species similar to those used in the current study(28). According to Okem et al. (29), the MIC values of less than $1 \mu \mathrm{g} / \mathrm{ml}$ of extracts and isolated compounds is referred as potent, while other authors report isolated compounds to be highly active and most potent at $12.5 \mu \mathrm{g} / \mathrm{ml}$ against pathogenic Mycobacterium species $(30,31)$. The selected $M$. carbono (Tabela 1), sub-espetro, DEPT-90, apresenta cinco carbonos metano $(\mathrm{C}-\mathrm{H})$ enquanto que DEPT-135 sugere dez metilenos $\left(\mathrm{CH}_{2}\right.$ negativo), sete metilos $\left(\mathrm{CH}_{3}\right.$, positivo) e oito grupos de carbonos quaternários, a espectroscopia de massa de impacto de eletrões indica um ião molecular $[\mathrm{M}-\mathrm{H}]^{+}$a m/z 457,7; que está em concordância com a massa molecular de $456,7 \mathrm{~g} \mathrm{~mol}^{-1}$ do ácido oleanólico.

\section{Ácido 3-O-Acetil-Oleanólico (OAA)}

Foi obtido um pó branco após recristalização com metanol, o ponto de fusão observado foi entre $261-263{ }^{\circ} \mathrm{C}$, o espetro de infravermelhos mostra absorção a 3562 $\mathrm{cm}^{-1}$ indicando assim um alongamento da ligação hidroxilo $(\mathrm{O}-\mathrm{H})$, o sinal a $1767 \mathrm{~cm}^{-1}$ é resultado de um grupo carbonilo $(\mathrm{C}=\mathrm{O}$, éster $)$, enquanto que o sinal a 1729 $\mathrm{cm}^{-1}$ é devido à presença de um alargamento do grupo carbonilo $(\mathrm{C}=\mathrm{O}$, ácido), estes dados provavelmente confirmam a presença de um grupo funcional carboxilo (COOH). O sinal a $2921 \mathrm{~cm}^{-1}$ é devido ao alongamento de um alcano altamente saturada $(\mathrm{C}-\mathrm{H})$. O desacoplamento de protão no espetro ${ }^{13} \mathrm{C}$-NMR mostra 32 átomos de carbono (Tabela 1), sub-espetro DEPT-90 mostra cinco metanos $(\mathrm{CH})$ enquanto que o DEPT-135 sugere a presença de 10 metilenos ( $\mathrm{CH}_{2}$ negativo), sete metilos $\left(\mathrm{CH}_{3}\right.$, positivo) e oito grupos com carbonos quaternários. A espectroscopia de massa de impacto de eletrões indica um ião molecular $[\mathrm{M}-\mathrm{H}]^{+}$a m/z 499,37 o que está em concordância com a massa molecular de 498,37 $\mathrm{g} \mathrm{mol}^{-1}$ do acetato de ácido oleanólico (OAA).

Diversa literatura encontra-se disponível relativamente às atividades biológicas do $\mathrm{OA}$, incluindo atividade anti-inflamatória, antioxidante, anti-HIV, antibacteriana, tripanocida, doenças cardiovasculares, cancro, aterosclerose e diabetes (27). Neste estudo atual, o maior foco foi em usar o OA para sintetizar e desenvolver compostos farmacologicamente ativos para diversos alvos utilizando ingredientes naturais contra as doenças mais comuns relacionadas com deficiência imunitária crónica. Neste trabalho, o OA exibiu uma potente atividade antimicobacteriana de $0,042\left(\mathrm{IC}_{50}\right.$ in $\left.\mu \mathrm{g} / \mathrm{ml}\right)$ sendo duas vezes mais eficaz que o seu derivado OAA. Este resultado está concordante com outros triterpenos tais como o ácido betulinico, betulina, lupenona, e betulona provenientes da Alnus incana (Betulaceae) que apresentaram um $\mathrm{IC}_{50}$ variando entre $2,4 \mathrm{a}>100 \mu \mathrm{g} / \mathrm{ml}$ contra espécies similares àquelas utilizadas neste estudo (28). De acordo com Okem et al. (29), os valores de MIC inferiores a $1 \mu \mathrm{g} / \mathrm{mL}$ de extratos e compostos isolados é referido como potente, enquanto que outros autores reportam que os compostos isolados são altamente ati- 
tuberculosis strain was more susceptible to rifampicin $\left(\mathrm{MIC}_{99}\right.$ of $\left.0.01 \mu \mathrm{g} / \mathrm{ml}\right)$ than kanamycin $\left(\mathrm{MIC}_{99}\right.$ of $3.125 \mu \mathrm{g} / \mathrm{ml}$ ). Although OA is known to possess poor solubility in most solvents, it can be used as a model template for the synthesis of potent drugs that can be used in the treatment of devastating human pathogens. Earlier, OA from other plant sources revealed potent antimicrobial activity (32), while extracts from $S$. aromaticum flower buds exhibited potent antibacterial activity against important pathogens including Escherichia coli, Klebsiella pneumonia, Pseudomonas aeruginosa, Proteus vulgaris, Campylobacter jejuni, Salmonella enteritis, Bacillus subtilis, Staphylococcus aureus, Staphylococcus epidirmidis, among others, and antifungal activity against Candida albicans, Aspergillus niger, Trichophyton mentagrophytes and Saccharomycetes cerevisiae $(33,34)$. Other important biological activities of $S$. aromaticum include modulatory effects, increased sexual activity, increased sperm count, antioxidant activity, and enhanced learning memory and recall (35-41). Furthermore, OA from other plants exhibited potent anti-platelet activity (42).

3-O-Acetyl-Oleanolic acid exhibited high antiplasmodial activity $\left(\mathrm{IC}_{50}\right.$ of $4.3 \mu \mathrm{g} / \mathrm{ml}$ ) against P. falciparum (Table 2). Although Pillay et al. (43) reported the $\mathrm{IC}_{50}$ values of some compounds ranging from 0.4 to $70 \mu \mathrm{g} / \mathrm{ml}$ against related strains, the antiplasmodial activity of the OAA derivative in our study is related with those of Simelane et al. (44), who reported the derivative of the isolated compound to possess better anti-plasmodial activity compared to the isolated compound. The selected strain was more susceptible to artesunate than chloroquine.

Considering the phytochemical point of view, besides the newly isolated oleanolic acid, the flower buds of Syzygium aromaticum revealed the presence of eugenol, caryophyllene, eugenyl acetate as the major compounds $(45,46)$. In the current work, the two compounds revealed $\mathrm{IC}_{50}$ of $>300 \mu \mathrm{g} / \mathrm{ml}$ against the human embryonic kidney and the human hepatocellular carcinoma cell lines. However, according to Sahranavard et al. (47), the isolated compounds are considered toxic when exhibiting $\mathrm{LD}_{50}$ values of about $100 \mu \mathrm{g} / \mathrm{ml}$ or less. Some isolated compounds and plant extracts from plant materials reportedly exhibited very low $\mathrm{IC}_{50}$, hence, they are toxic (48). vos e mais potentes a $12,5 \mu \mathrm{g} / \mathrm{ml}$ contra espécies patogénicas Mycobacterium (30,31). A estirpe selecionada M. tuberculosis apresentava maior suscetibilidade para a rifampicina ( $\mathrm{MIC}_{99}$ of $0,01 \mu \mathrm{g} / \mathrm{mL}$ ) do que para a canamicina ( $\mathrm{MIC}_{99}$ of $3,125 \mu \mathrm{g} / \mathrm{mL}$ ). Apesar de OA ser conhecido por ter baixa solubilidade na maioria dos solventes poderá ser utilizado como modelo para a síntese de fármacos potentes, que poderão ser utilizados no tratamento de patogénicos humanos. Outros estudos, descrevem OA obtido de outras plantas, e que apresentou uma potente atividade antimicrobiana (32), enquanto que os extratos de botões de flores de $S$. aromaticum exibiram uma atividade antibacteriana potente contra patógenos importantes incluindo Escherichia coli, Klebsiella pneumonia, Pseudomonas aeruginosa, Proteus vulgaris, Campylobacter jejuni, Salmonella enteritis, Bacillus subtilis, Staphylococcus aureus, S. epidirmidis entre outras, e atividade antifúngica contra Candida albicans, Aspergillus niger, Trichophyton mentagrophytes and Saccharomycetes cerevisiae $(33,34)$. Outras atividades biológicas importantes do $S$. aromaticum incluem o seu efeito modulatório, aumento da atividade sexual, aumento da contagem de espermatozoides, atividade antioxidante e memoria de aprendizagem melhorada (35-41). Para além disso, o OA proveniente de outras plantas exibiu uma atividade anti plaquetária potente (42).

O Ácido 3-O-Acetil-Oleanólico exibiu uma boa atividade antiplasmódica $\left(\mathrm{IC}_{50}\right.$ de $\left.4,3 \mu \mathrm{g} / \mathrm{ml}\right)$ contra $P$. falciparum (Tabela 2). Apesar de Pillay et al. (43) ter reportado $\mathrm{IC}_{50}$ de alguns compostos variando de 0,4 até $70 \mu \mathrm{g} / \mathrm{ml}$ contra uma estirpe relacionada, a atividade antiplasmódica do derivado OAA no nosso estudo, relaciona-se com os resultados de Simelane et al. (44), que reportou que o derivado possui melhor atividade antiplasmódica do que o composto isolado. A estirpe selecionada era mais suscetível ao artenusato do que à cloroquina.

Do ponto de vista fitoquimico, além do ácido oleanólico isolado, os botões das flores de Syiuzygm aromaticum revelaram a presença de eugenol, cariofileno, acetato de eugenil como compostos maioritários $(45,46)$. No corrente trabalho, os dois compostos revelaram um $\mathrm{IC}_{50}$ de $>300 \mu \mathrm{g} / \mathrm{ml}$ contra as linhas celulares renais embrionárias humanas e do carcinoma hepatocelular humano. No entanto, de acordo com Sahranavard et al. (47), os compostos isolados são referidos como tóxicos quando exibem $\mathrm{LD}_{50}$ de cerca $100 \mu \mathrm{g} / \mathrm{ml}$ ou menos. Os compostos isolados e extratos de plantas que exibem $\mathrm{IC}_{50}$ muito baixos, são considerados tóxicos (48). 


\section{Conclusion}

Physical and biological properties were altered with the introduction of a lipophilic acetyl group in replacement of the hydroxyl group in OA. The anti-malaria activity against $P$. falciparum of the derivative OAA is observed to be higher than that of $\mathrm{OA}\left(\mathrm{IC}_{50} \mathrm{OAA}=4.3 \mu \mathrm{g} / \mathrm{ml}\right.$, $\mathrm{IC}_{50} \mathrm{OA}=27.4 \mu \mathrm{g} / \mathrm{ml}$ ). Moreover, the results suggest that the compounds showed no cytotoxicity at low to moderate concentrations against the cell lines used. Although the antimycobacterial potential of $\mathrm{OA}$ is better than the derivative OAA, the construction of a lead molecule for the treatment of malaria may be obtained by converting $\mathrm{OA}$ to an ester. The release of the position C-28 to react with several other anti-malarial molecules can be accomplished using click chemistry, for example. The hydrolysis of the ester at the $\mathrm{C}-3$ position to a hydroxyl group can then be performed using mild alkaline conditions such that more derivatives can be designed using OA as starting material to obtain future antiplasmodial compounds.

\section{Acknowledgments}

We acknowledge the financial contribution of University of Zululand Research committee.

\section{Authors' Contributions}

VOF, ARNI, OM and MS, methodology and research; PR supervision and ARP supervision and final review.

\section{Conflict of Interest}

The senior editor co-authoring this manuscript had no participation in the review nor in the decision process.

All authors have declared there were no financial and/ or personal relationships that may present a potential conflict of interest.

\section{Conclusão}

As propriedades fisicas e biologicas foram alteradas com a introdução de um grupo acetil lipofílico em substituição de um grupo hidroxilico no OA. Observou-se que a atividade antimalária contra $P$. falciparum do derivado $\mathrm{OAA}$ foi superior à do $\mathrm{OA}\left(\mathrm{IC}_{50} \mathrm{OAA}=4,3 \mu \mathrm{g} /\right.$ $\left.\mathrm{ml}, \mathrm{IC}_{50} \mathrm{OA}=27,4 \mu \mathrm{g} / \mathrm{ml}\right)$. Os resultados sugerem também que os compostos não apresentam citotoxicidade a concentrações baixas a moderadas, em relação às linhas celulares utilizadas. Embora o potencial antimicobacteriano de OA seja superior ao derivado OAA, a construção de uma molécula protótipo, para o tratamento da malária, pode ser obtida pela conversão de $\mathrm{OA}$ em um éster. A libertação da posição C-28 para reagir com várias outras moléculas antimaláricas, pode ser realizada, por exemplo, usando a "click chemistry".

Então a hidrólise do éster na posição C-3 no grupo hidroxilo, usando condições alcalinas suaves, pode ser realizada e mais derivados podem ser preparados, usando OA como material de partida, para obter futuros compostos para o tratamento da malária.

\section{Agradecimentos}

Agradecemos o apoio financeiro recebido da Comissão de Investigação da University of Zululand.

\section{Contribuição dos Autores}

VOF, ARNI, OM e MS, metodologia, investigação; PR, supervisão e ARP, supervisão e revisão final.

\section{Conflito de Interesses}

O editor senior envolvido na autoria deste manuscrito não teve qualquer participação no processo de revisão ou de decisão.

Todos os autores declararam não haver relações financeiras e/ou pessoais que possam representar um potencial conflito de interesses. 


\section{References / Referências}

1. Raj R, Biot C, Carrere-Kremer S, Kremmer L, Guerardel Y, Gut J, Rosenthal PJ, Forge D, Kumar V. 7-Chloroquinoline-isatin conjugates: Anti-malarial, anti-tubacular, and cytotoxicity evaluation. Chem Biol Drug Des.. 2014; 83:622-629.

2. Gurcha SS, Usha V, Cox JAG, Fütterer K, Abrahams KA, Bhatt A, Alderwick LJ, Reynolds RC, Loman NJ, Nataraj V, Alemparte C, Barros D, Lloyd AJ, Ballell L, Hobrath JV, Besra GS. Biochemical and Structural Characterization of Mycobacterial Aspartyl-tRNA Synthetase AspS, a promising TB Drug Target. PLoS ONE. 2014; 9(11): e113568. doi:10.1371/journal.pone.0113568.

3. Adebayo JO, Krettli AU. Potential antimalarials from Nigerian plants: A review. J Ethnopharmacol. 2011; 133: $289-302$.

4. Wongsrichanalai C, Varma JK, Juliano JJ, Kimerling ME, MacArthur JR. Extensive drug resistance in malaria and tuberculosis. Emerg Infect Dis. 2010; 16(7):1063-1067.

5. Mohammad A. A review of antimycobacterial drugs in Development. Mini Rev Med Chem. 2012; 12(13):1404-1418.

6. Nzila A, Ma Z, Chibale K. Drug repositioning in the treatment of malaria and TB. Future Med Chem. 2011; 3(11):1413-1426.

7. Bates I, Fenton C, Gruber J, Lalloo D, Lara AM, Squire SB, Theobald S, Thomson R, Tolhurst R Vulnerability to malaria, tuberculosis, and HIV/AIDS infection and disease. Part II: determinants operating at environmental and institutional level. Lancet Infect Dis. 2004; 4:368-375

8. Aparna Y, Kranthi SU, Sarada J. Syzygium aromaticum - A common food spice with potential quorom quenching activity on Serratia sps YAJS. Int J Curr Microbiol Appl Scis. 2014; 3(7):93-102.

9. Bhowmik D, Kumar KPS, Yadav A, Srivastava S, Paswan S, Dutta AS. Recent trends in Indian traditional herbs Syzygium aromaticum and its health benefits. J. Pharmacogn. Phytochem. 2012; 1(1):13-22.

10. Aiko V, Mehta A. Inhibitory effect of clove (Syzygium aromaticum) on the growth of Penicillium citrinum and citrinin production. J Food Safety. 2013; 33:440-444.

11. Yazdanpanah L, Mohamadi N (2014). Antifungal activity of the clove essential oil from Syzygium aromaticum on Paecilomycetes variotii agent of pistachio dieback. J Biodivers Environ Sci. 2014; 4(6):42-45

12. Avasthi S, Gautam AK, Bhadauria R. Antifungal activity of plant products against Aspergillus niger: A potential application in the control of a spoilage fungus. Biol Forum. 2010; 2(1):53-55.

13. Khewkhom N, Shangchote S. Postharvest antifungal activity of extracts and compounds from Cinnamomum zeynalucum, Boesenbergia pandurata and Syzygium aromaticum against Colletotrichum gloeosporioides and Botryodiplodia theobromae. AJOFAI. Special Issue: 2009; 125-132

14. Pandey A, Singh P. Antibacterial activity of Syzygium aromaticum (clove) with metal ion effect against food borne pathogens. Asian J. Plant Sci. Res. 2011; 1(2):69-80.

15. Saeed M, Nadeem M, Khan MR, Shabir MA, Shehzad A, Amir RM . Antimicrobial activity of Syzygium aromaticum extracts against food spoilage bacteria. Afr. J. Microbiol. Res. 2013; 7(41):4848-4856.

16. Dua A, Garg G, Nagar S, Mahajan R. Methanol extract of clove (Syzygium aromaticum Linn) damages cells and inhibits growth of enteropathogens. J. Innov. Biol. 2014; 1(4):200-205.

17. Srivastava K, Sherenda S, Mishra SK, De K. In vitro antimicrobial activity and phytochemical screening of Syzygium aromaticum. Asian J. Res. Pharm. Sci. 2014; 4(1):12-15.

18. Cortés-Rojas DF, de Souza CRF, Oliveia WP. Clove (Syzygium aromaticum): A precious spice.Asian Pac. J. Trop. Biomed. 2014; 4(2):90-96.

19. Milind P, Deepa K. Clove: A champion spice. Int J Res Ayurveda Pharm. 2011; 2(1):47-54.

20. Collins, L.A., Franzblau, S.G. Microplate Alamar Blue assay versus BACTEC 460 for high-throughput screening of compounds against Mycobacterium tuberculosis and Mycobacterium avium. Antimicrob Agents Chemother. 1997; 41:1004-1009.

21. Birdi T, D'souza D, Tolani M, Daswani P, Nair V, Tetali P, Toro JC, Hoffner S (2002). Assessment of the activity of selected Indian medicinal plants against Mycobacterium tuberculosis: A preliminary screening using the microplate Alamar Blue Assay. European J Med Plants. 2002; 2(4):308-323.

22. Trager W, Jensen JB. Human malaria parasite in continuous culture. Science. 1976; 193: 673-675.

23. Makler MT, Ries JM, Williams JA. Parasite lactate dehydrogenase as an assay for Plasmodium falciparum drug sensitivity. Am J Trop Med Hyg. 1993; 48(6):739-741.

24. Mosma T. Rapid coloricmetric assay for cellular growth and survival: Application to proliferation and cytotoxicity assays. J. Immunol. Methods. 1983; 65: 55-65.

25. Soica C, Oprean C, Borcan F, Danciu C, Trandafirescu C, Coricovan D, Cráiniceanu Z, Dehelean CA, Munteanu M.The synergistic biologic activity of oleanolic and ursolic acids in complex with hydroxypropyl-ץ-cyclodextrin. Molecules. 2014; 19:4924-4940.

26. Liu J . Pharmacology of oleanolic acid and ursolic acid. J Ethnopharmacol 1995; 49:57-68.

27. Paszel-Jaworska A, Romaniuk A, Rybczynska R. Molecular mechanisms of biological activity of oleanolic acid - a source of inspiration for a new drugs design. Mini Rev Org Chem. 2015; 11(3): 330-342.

28. Li H, Webster D, Johnson JA, Gray CA. Antimycobacterial triterpenes from the Canadian medicinal plant Alnus incana. J Ethnopharmacol 2015; 165:148-151.doi: 10.1016/j.jep.2015.02.042

29. Okem A, Finnie JF, van Staden J . Pharmacological, genotoxic and phytochemical properties of selected South African medicinal plants used in treating stomach-related ailments. J Ethnopharmacol 2011; 139:712-720. doi: 10.1016/j.jep.2011.11.034

30. Newton SM, Lau C, Gurcha SS, Besra GS, Wright CW. The evaluation forty-three plant species for in vitro antimycobacterial activities; isolation of active constituents from Psoralea corylifolia and Sanguinaria canadensis. J Ethnopharmacol 2002; 79(1):57-67. doi: 10.1016/s0378-8741(01)00350-6.

31. León-Díaz R, Meckes M, Said-Fernández S, Molina-Salinas GM, Vargas-Villarreal J, Torres J, Luna-Herrera J, Jiménez-Arellanes A. Antimycobacterial neolignans isolated from Aristolochia taliscana. Mem Inst Oswaldo Cruz, Rio de Janeiro. 2010; 105(1): 45-51. 
32. Jesus JA, Lago JH, Laurenti MD, Yamamoto ES, Passero LF. Antimicrobial activity of oleanolic and ursolic acids: an update. Evid Based Complement Alternat Med. 2015;2015:620472. doi:10.1155/2015/620472.

33. Menghani E, Rana A, Saraswat P, Pareek A. Antibacterial potentials of two Indian spices.Int J Pharma Bio Sci. 2014; 5(3):666-672.

34. Pulikottil SJ, Nath S . Potential of clove of Syzygium aromaticum in development of a therapeutic agent for periodontal disease: A review. S. Afr. Dent. J. 2015; 70(3):108-115.

35. Chikere OU, Liman FL, Nnnabuihe ED, Uchechi EE, Jesse AC. The effects of methanolic extract of Syzygium aromaticum (clove bud) on the histology of testis in adult male Wistar rats. J. Nat. Sci. Res. 2015; 5(12):1-6.

36. Sharma A, Kumar M, Kaur S. Modulatory effects of Syzygium aromaticum (L.) Merr \& Perry and Cinnamomum tamala Nees \& Ebrem on the toxicity induced chromium trioxide. Phytopharmacology 2011; 1(4):71-81.

37. Tajuddin SA, Abdul L, Iqbal AQ. Effect of 50\% ethanolic extract of Syzygium aromaticum (L.) Merr \& Perry (Clove) on sexual behavior of normal male rats. BMC Complement Altern Med.. 2004; 4:17 doi:10.1186/1472-6882/4/17.

38. Dashti-R MH, Morshedi A. The effect of Syzygium aromaticum (clove) on learning memory in mice. Asian J. Tradit. Med. 2009; 4(4):128-133.

39. Ramadan MF, Asker MMS, Tadros M. Lipid profile, antiradical power and antimicrobial properties of Syzygium aromaticum oil. Grasas Y. Aceites. 2013; 4(5):509-520.

40. Devi SA, Umasankar ME, Babu S. A comparative study of antioxidants properties in common Indian spices. Int. Res. J. Pharm 2012; 3(5):465-468.

41. Adefegha SA, Oboh G. Water extractable phytochemicals from Nigerian spices inhibit $\mathrm{Fe}^{2+}$ induced lipid peroxidation in rat brain in vitro. Food Process Technol. 2011; doi: 10.4172/2157-7110.1000104

42. Babalola IT, Shode FO, Aledakun EA, Opoku AR, Mosa RA. Platelet-aggregation inhibitory activity of oleanolic acid, ursolic acid, betulinic acid, and maslinic acid. J Pharmacogn Phytochem. 2013; 1(6):54-60.

43. Pillay P, Vleggar R, Maharaj VJ, Smith PJ, Lategan CA. Isolation and identification of antiplasmodial sesquiterpene lactones from Oncosiphon piluliferum. J Ethnopharmacol. 2007; 112:71-76. doi: 10.1016/j.jep.2007.02.002

44. Simelane BC, Shonhai A, Shode FO, Smith P, Singh M, Opoku AR. Anti-plasmodial activity of some Zulu medicinal plants and of some triterpenes isolated from them. Molecules. 2013; 18:12313-12323.

45. Nassar MI, Gaara AH, El-Ghorab AH, Farrag ARH, Shen H, Huq E, Mabry TJ . Chemical constituents of cloves (Syzygium aromaticum, Myrtaceae) and their antioxidant activity. Rev Latinoamer Quim. 2015; 35(3):47-57.

46. Gupta M, Singh D, Gularia P, Gupta S. GCMS analysis and identifications of chemical constituents of Syzygium aromaticum, Brassica compestris, and cow ghee. J Chem Pharm Res. 2015; 7(1):568-572.

47. Sahranavard S, Naghibi F, Mosaddegh M, Esmaeili S, Sarkhail P, Taghvaei M . Cytotoxic activities of selected medicinal plants from Iran and phytochemical evaluation of the most potent extract. Res Pharm Sci. 2009; 4(2): 133-137.

48. Mthethwa NS, Oyedeji BAO, Obi CL, Aiyegoro OA. Anti-staphylococcal, anti-HIV and cytotoxicity studies of four South African medicinal plants and isolation of bioactive compounds from Cassine transvaalensis (Burtt. Davy) codd. BMC Complement Altern Med. 2014; 14:512. doi: 10.1186/1472-6882-14-512 